\title{
Natural Approach to Coexisting Non-alcoholic Fatty Liver Disease and Polycystic Ovarian Syndrome Xianqin Qu*
}

School of Medical \& Molecular Biosciences, University of Technology Sydney, NSW 2007, Australia

\begin{abstract}
Polycystic Ovarian Syndrome (PCOS) is a common reproductive and metabolic disorder. It is considered the ovarian manifestation of metabolic syndrome and is a common cause for an ovulatory infertility. PCOS commonly coexists with other metabolic manifestations such as Non-alcoholic Fatty Liver Disease (NAFLD) which leads to therapeutic challenges especially when infertility is involved. Existing treatment strategies for PCOS have limited success, side effects or are costly giving rise to a curiosity in women to seek alternative therapies such as Chinese Medicine. Chinese medicine formulae with multiple natural ingredients may have beneficial endocrine, cardio metabolic and reproductive effects offering an optimal treatment approach for women with NAFLD, PCOS and infertility.
\end{abstract}

Keywords: Polycystic ovarian syndrome; Nonalcoholic fatty liver disease; Infertility; Chinese herbal medicine; Clinical trial

\section{Introduction}

Polycystic Ovarian Syndrome (PCOS) is a common disorder affecting up to $21 \%$ of women of childbearing age [1]. Initially thought to be primarily a reproductive disorder, recent findings suggest that $50-80 \%$ of women with PCOS are obese and have insulin resistance, a hallmark of metabolic syndrome [2]. It therefore seems appropriate to consider PCOS as the ovarian manifestation of metabolic syndrome. Similarly, Nonalcoholic Fatty Liver Disease (NAFLD) has been defined as the hepatic manifestation of metabolic syndrome. Clinically, PCOS commonly coexists with NAFLD [3], which further complicates the therapeutic approach, especially when infertility is of concern.

Conventional approaches to PCOS with infertility have been well documented in the literature [4,5], including lifestyle modifications and the use of metformin either alone or in combination with clomiphene, gonadotropins, ovarian drilling or assisted reproductive techniques. However, there are few studies for management of the complex of hyperglycaemia, NAFLD and PCOS with infertility. Alongside this, the combination of NAFLD and PCOS impedes treatment with some of the chemical pharmacotherapies available when there is liver damage and impaired liver function [6]. The clinical features of PCOS associated with NAFLD are heterogeneous and vary in intensity. The treatment for the combination of NAFLD and PCOS with infertility therefore presents a therapeutic challenge. A recent report suggests that women with a diagnosis of NAFLD prior to giving birth have increased risks for adverse pregnancy outcomes, including gestational diabetes, preeclampsia, caesarean section and preterm birth [7]. It is this context along with the increase in prevalence of coexisting PCOS and NAFLD that often gives rise to a curiosity in women to seek alternative therapies, especially for those who are desirous to achieve conception naturally. Over the last ten years, this condition has become one of the most treated disorders in our Chinese medicine clinic with much success.

Chinese medicinal herbs have been used for many years to treat gynaecological disorders according to Traditional Chinese Medicine (TCM) theory. In the last decades, experimental and clinical studies have shown that these herbal ingredients could regulate gonadotropinreleasing hormone to induce ovulation and promote blood flow and microcirculation to the ovaries and uterus to improve their function $[8,9]$. Zhang et al. have summarized four randomized controlled trials that demonstrated a synergetic effect of $\mathrm{CHM}$ on clomiphene for PCOS with subfertility [10]. We have also observed that most patients with PCOS and NAFLD present with a 'blood stasis syndrome' in TCM which is related to hyperlipidaemia and a prethrombotic state.
Therefore, our treatment strategies for PCOS and NAFLD initially focuses on ameliorating hyperlipidaemia and decreasing thrombotic factors by using herbs such as Radix Salvia miltiorrhizae, Fructus Crataegus pinnatifida, Fructus Gardenia florida, Radix Angelicae sinensis and Rhizoma Coptis chinensis.

Herbal medicines, such as Fructus Gardenia florida, Fructus Lycium barbarum and Rhizoma Coptis chinensis, have also demonstrated a beneficial effect on insulin resistance and glucose intolerance [1113]. This is particularly relevant when hyperlipidaemia and NAFLD implicates liver damage as the use of drugs such as statins and metformin are subsequently cautioned. Given that NAFLD significantly contributes to adverse outcomes of pregnancy in women with PCOS, Salvia miltiorrhizae and Fructus Gardenia florida are important ingredients for PCOS women with NAFLD because the active compounds, such as tanshinone IIA and gypenoside, lower serum cholesterol and triglycerides and also prevents ectopic fat deposition in the liver [14]. Oxidative stress plays a role in both liver damage and ovarian dysfunction in NAFLD and PCOS respectively [15]. Naturally occurring compounds in the formulae, such as berberine and polysaccharides, improve clinical outcomes which may be through their antioxidant activity $[16,17]$.

Furthermore, as herbal formulae contain many different ingredients, the effect is often multi-targeted. In my clinical practice it has become evident that treating metabolic manifestations simultaneously achieves results more quickly than simply targeting one aspect, in the way that monotherapies operate. Chinese medicine formulae with multiple natural ingredients is capable of producing beneficial endocrine, cardiometabolic, and reproductive effects offering an optimal treatment approach for women with NAFLD, PCOS and infertility. Additionally herbal medicines have fewer side effects compared to standard drug therapies. Despite these benefits, most studies for PCOS are small, nonrandomized or uncontrolled and there is no clinical study reported

*Corresponding author: Xianqin Qu, School of Medical Molecular \& Biosciences, University of Technology Sydney, NSW 2007, Australia, Tel: +61-2-9514 7852; Fax: +61-2-9514 8206; E-mail: Xianqin.Qu@uts.edu.au

Received June 08, 2015; Accepted July 01, 2015; Published July 10, 2015

Citation: Qu X (2015) Natural Approach to Coexisting Non-alcoholic Fatty Liver Disease and Polycystic Ovarian Syndrome. Endocrinol Metab Synd 4: 182. doi:10.4172/2161-1017.1000182

Copyright: () $2015 \mathrm{Qu} \mathrm{X.} \mathrm{This} \mathrm{is} \mathrm{an} \mathrm{open-access} \mathrm{article} \mathrm{distributed} \mathrm{under} \mathrm{the}$ terms of the Creative Commons Attribution License, which permits unrestricted use, distribution, and reproduction in any medium, provided the original author and source are credited. 
for CHM treatment for the combination of NAFLD and PCOS with infertility. This case report presents the use of CHM to target multiple abnormalities, firstly focusing on hyperlipidaemia and impaired liver function while inducing menstruation for amenorrhea and subsequently shifting to focus on improving ovarian function and fertility. Although the evidence is limited by one case, successful treatment with $\mathrm{CHM}$ for this case highlights the potential of natural therapy to manage coexisting NAFLD and PCOS cohesively whilst promoting fertility.

In summary, co-existing NAFLD and PCOS with infertility presents a therapeutic challenge for both pharmaceutical medication and natural therapy. A uniform treatment for this complex metabolic, endocrinal and reproductive disorder does not exist. To achieve successful conception and reduce adverse outcomes of pregnancy, the best-tailored treatment with CHM should be applied after an accurate evaluation of patients' characteristics. Meanwhile, randomized and controlled multicenter trials should be performed to establish evidence-based CHM therapy for the treatment of NAFLD and PCOS with infertility.

\section{References}

1. Shorakae S, Boyle J, Teede H (2014) Polycystic ovary syndrome: a common hormonal condition with major metabolic sequelae that physicians should know about. Intern Med 44: 720-726.

2. Rahmanpour H, Jamal L, Mousavinasab S, Esmailzadeh A, Azarkhish K (2012) Association between polycystic ovarian syndrome, overweight, and metabolic syndrome in adolescents. J Pediatr Adolesc Gynecol 25: 208-212.

3. Vassilatou E (2014) Nonalcoholic fatty liver disease and polycystic ovary syndrome. World J Gastroenterol 20: 8351-8363.

4. Berger JJ, Bates GW Jr (2014) Optimal management of subfertility in polycystic ovary syndrome. Int J Wom Health 6: 613-621.

5. Rocca ML, Venturella R, Mocciaro R, Di Cello A, Sacchinelli A, et al. (2015) Polycystic ovary syndrome: chemical pharmacotherapy. Expert Opin Pharmacother 16: 1369-1393.
6. Kinnman N, Hultcrantz R (2001) Lipid lowering medication and hepatotoxicity. Intern Med 250: 183-185.

7. Hagström H, Höijer J, Ludvigsson J.F, Bottai M, Ekbom A,et al. (2015) Adverse outcomes of pregnancy in women with non-alcoholic fatty liver disease. Liver Int, doi: 10.1111/liv.12902. [Epub ahead of print].

8. Reid K, Stuart K (2011) Efficacy of Traditional Chinese Herbal medicine in the management of female infertility: a systematic review. Complement Ther Med 19: 319-331.

9. Huang S.T, Chen A.P (2008) Traditional Chinese medicine and infertility. Curr Opin Obstet Gynecol 20: 211-215.

10. Zhang J, Li T, Zhou L, Tang L, Xu L, et al. (2010) Chinese herbal medicine for subfertile women with polycystic ovarian syndrome. Cochrane Database Sys Rev 8: 1-45.

11. Ji W, Gong BQ (2008) Hypolipidemic activity and mechanism of purified herbal extract of Salvia miltiorrhiza in hyperlipidemic rats. J Ethnopharmacol 119: 291 298

12. Tan Y, Kamal MA, Wang ZZ, Seale JP, Qu X, et al. (2011) Chinese herbal extracts (SK0506) as a potential candidate for the therapy of metabolic syndrome. Clin Sci 120: 297-305

13. Li Y, Ma H, Zhang Y, Kuang H, Ng E, et al. (2013) Effect of berberine on insulin resistance in women with polycystic ovary syndrome: study protocol for a randomized multicenter controlled trial. Trials, 14: 226.

14. Tan Y, Lao W, Xiao L, Seale J, Qu X, et al. (2013) Managing the Combination of Nonalcoholic Fatty Liver Disease and Metabolic Syndrome with Chinese Herbal Extracts in High-Fat-Diet Fed Rats. Evid Based Complement 2013: $1-10$.

15. Kurdoglu Z, Ozkol H, Tuluce Y, Koyuncu I (2012) Oxidative status and its relation with insulin resistance in young non-obese women with polycystic ovary syndrome. J Endocrinol Invest 35: 317-321.

16. Jang MH, Kim HY, Kang KS, Yokozawa T, Park JH (2009) Hydroxyl radical scavenging activities of isoquinoline alkaloids isolated from Coptis chinensis. Arch Pharm Res 32: 341-345.

17. Luo Q, Cui X, Yan J, Yang M, Liu J, et al. (2011) Antagonistic effects of Lycium barbarum polysaccharides on the impaired reproductive system of male rats induced by local subchronic exposure to 60Co-y irradiation. Phytother Res 25 694-701.
Citation: Qu X (2015) Natural Approach to Coexisting Non-alcoholic Fatty Liver Disease and Polycystic Ovarian Syndrome. Endocrinol Metab Synd 4: 182 doi:10.4172/2161-1017.1000182
Submit your next manuscript and get advantages of OMICS Group submissions

Unique features:

User friendly/feasible website-translation of your paper to 50 world's leading languages

Audio Version of published paper

Digital articles to share and explore

Special features:

350 Open Access Journals

30,000 editorial team

21 days rapid review proces

Quality and quick editorial, review and publication processing

Indexing at PubMed (partial), Scopus, EBSCO, Index Copernicus and Google Scholar etc

Sharing Option: Social Networking Enabled

Authors, Reviewers and Editors rewarded with online Scientific Credits

Better discount for your subsequent articles

Submit your manuscript at: http://www.editorialmanager.com/biochem 